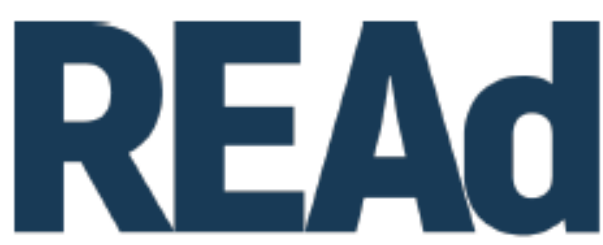

Revista Eletrônica de Administração

\title{
A COORDENAÇÃO DE ESFORÇOS COLETIVOS PARA ENFRENTAR A PANDEMIA DO NOVO CORONAVÍRUS: UM ESTUDO DE CASO SOBRE O HACKATHON HACK FOR BRAZIL | COVID-19 1
}

\author{
Jorge Renato Verschoore ${ }^{2}$
}

http://dx.doi.org/10.1590/1413-2311.283.103388

\section{RESUMO}

Este artigo se alinha aos esforços da comunidade acadêmica para lidar com as consequências da Covid-19. O estudo se debruça sobre a iniciativa Hack for Brazil, hackathon cívico que reuniu mais de mil participantes para investigar como a coordenação de esforços coletivos é realizada em contextos de grande escala e intenso dinamismo. Alicerçado nos fundamentos da microcoordenação de redes, o artigo analisou cinco construtos: papéis, perfis, envolvimento, interação e alinhamento. As evidências foram coletadas por meio de documentos, entrevistas individuais e de um grupo focal com participantes do hackathon. Os resultados evidenciaram que a microcoordenação de redes assume uma característica adaptativa e emergente em hackathons de grandes proporções. $\mathrm{O}$ intenso dinamismo impede o fortalecimento de vínculos sociais e dificulta a interação e o envolvimento dos participantes. A grande escala amplia a heterogeneidade de perfis e possibilita que papéis de coordenação sejam assumidos organicamente pelos participantes. $\mathrm{O}$ alinhamento ocorre no calor das decisões, requerendo a formalização de normas para reforçar a assertividade dos participantes. O estudo trouxe contribuições às práticas empregadas pelos promotores de hackathons ao destacar os construtos que afetam sua implementação e replicação. Ao final, o estudo aponta janelas de oportunidades para investigações da microcoordenação de redes em contextos dinâmicos.

Palavras-chave: Covid-19. Hackathon. Redes. Coordenação. Hack for Brazil

\section{COORDINATION OF COLLECTIVE EFFORTS TO TACKLE THE NEW CORONAVIRUS PANDEMIC: A CASE STUDY ON THE HACKATHON HACK FOR BRAZIL | COVID-19}

This article aligns with the academic community's efforts to deal with the consequences of Covid-19. The study addresses the Hack for Brazil initiative, a civic hackathon that brought

\footnotetext{
${ }^{1}$ Recebido em 25/5/2020, aceito em 10/8/2020.

${ }^{2}$ Universidade do Vale do Rio dos Sinos - Programa de Pós-Graduação em Administração e Programa de PósGraduação em Gestão e Negócios; Porto Alegre - RS (Brasil); http://orcid.org/0000-0001-7588-7871; jorgevf@unisinos.br.
} 
together more than a thousand participants, to investigate how the coordination of collective efforts is carried out in contexts of large scale and intense dynamism. Based on the networks micro-coordination research, the article analyzed five constructs: roles, profiles, involvement, interaction and alignment. Evidence was collected through documents, individual interviews and a focus group with hackathon participants. Results showed that network microcoordination has an adaptive and emerging characteristic in large hackathons. The intense dynamism refrains the strengthening of social bonds and hinders the interaction and involvement of the participants. The large scale increases the heterogeneity of profiles and allows coordinating roles to be evolved organically by the participants. Alignment occurs in the heat of decisions, requiring the formalization of rules to reinforce the decisiveness of the participants. The study brought contributions to the practices employed by hackathon promoters by highlighting the constructs that affect their implementation and replication. In the end, the study points out windows of opportunities for investigating network microcoordination in dynamic contexts.

Keywords: Covid-19. Hackathon. Networks. Coordination. Hack for Brazil

\section{COORDINACIÓN DE ESFUERZOS COLECTIVOS PARA ENFRENTAR LA PANDEMIA DEL NUEVO CORONAVIRUS: UN ESTUDIO DE CASO SOBRE EL HACKATÓN HACK FOR BRAZIL | COVID-19}

Este artículo se alinea con los esfuerzos de la comunidad académica para abordar las consecuencias de Covid-19. El estudio analiza la iniciativa Hack for Brazil, un hackatón cívico que reunió a más de mil participantes, para investigar cómo se lleva a cabo la coordinación de los esfuerzos colectivos en contextos de gran escala e intenso dinamismo. Basado en los fundamentos de la microcoordinación de redes, el artículo analizó cinco constructos: roles, perfiles, participación, interacción y alineación. La evidencia se recopiló a través de documentos, entrevistas individuales y de un grupo focal con participantes de hackathon. Los resultados mostraron que la microcoordinación de redes asume una característica adaptativa y emergente en grandes hackatones. El intenso dinamismo impide el fortalecimiento de los lazos sociales y dificulta la interacción y la participación de los participantes. La gran escala aumenta la heterogeneidad de los perfiles y permite que los participantes asuman orgánicamente los roles de coordinación. La alineación ocurre en el fragor de las decisiones, lo que requiere la formalización de reglas para reforzar la asertividad de los participantes. El estudio aportó contribuciones a las prácticas empleadas por los promotores de hackatones al destacar los constructos que afectan su implementación y replicación. Al final, el estudio señala oportunidades para investigar la microcoordinación de redes en contextos dinámicos.

Palabras clave: Covid-19. Hackathon. Redes. Coordinación. Hack for Brazil 


\section{INTRODUÇÃO}

A crise decorrente da pandemia da Covid-19 acarretou impactos socioeconômicos sem precedentes. Restrições de deslocamento, isolamento social, queda da atividade econômica e aumento das taxas de desemprego, entre outros, são desafios que, de forma simultânea e nessa magnitude, nunca foram confrontados pela humanidade. As dificuldades impostas pela pandemia à população são crescentes e os efeitos deletérios à saúde repercutem mais intensamente em médicos, enfermeiros, assistentes e demais profissionais que precisam lidar diretamente com as consequências do novo coronavírus.

O aumento da tensão nas unidades de saúde, relatado pela imprensa nacional, demonstra a angústia de lidar com uma doença de rápida propagação e sem cura iminente. Em reportagem publicada pelo jornal Folha de SP, os profissionais descrevem o clima tenso existente nas unidades de atendimento e as demonstrações de transtorno de ansiedade entre os pacientes como uma pandemia de saúde mental (FOLHA DE SP, 2020). Neste contexto, médicos, enfermeiros e assistentes têm relatado a sobrecarga de trabalho, a falta de equipamentos para proteção e o permanente receio de contaminar seus familiares. Com tudo isso, um olhar otimista permite lembrar que crises como esta abrem oportunidades para ações e soluções criativas.

Um projeto de arquitetos do Rio Grande do Sul é um bom exemplo de ações voluntárias criativas. Ao perceberem a precária situação das salas de descanso e dos espaços de convivência de profissionais de saúde que atuavam em hospitais e unidades de atendimento conveniados ao SUS, arquitetos gaúchos decidiram unir competências para executar espaços de descompressão. O projeto conectou 50 arquitetos e doadores de móveis e artefatos e, em poucos dias, viabilizou a implementação de $370 \mathrm{~m}^{2}$ de espaços de descompressão e descanso em nove hospitais conveniados ao SUS. Ao interagir com os profissionais da saúde, os arquitetos conseguiram também ajudar a adaptar os espaços dos hospitais para atender as necessidades de proteção requeridas pelo novo coronavírus.

Ainda mais interessante é o fato de o projeto arquitetos voluntários ter surgido e crescido em uma maratona de esforços online dedicada a encontrar soluções para minimizar os impactos do novo coronavírus. Denominada de Hack for Brazil | Covid-19, a iniciativa foi organizada por 40 instituições brasileiras e reuniu mais de mil participantes entre os dias 23 e 27 de março de 2020 (TECHTUDO, 2020). Outras maratonas online de caráter público, conhecidas como civic hackathons (JOHNSON; ROBINSON, 2014), logo se seguiram no 
Brasil. O Hack for Good buscou soluções de comunicação sobre o novo coronavírus, o Mega Hack Covid-19 reuniu esforços para ajudar os pequenos negócios (PEGN, 2020) e o Hack pelo Futuro focou em soluções tecnológicas para a vida pós-pandemia (G1 PR, 2020).

Recorrer à sabedoria das multidões e aos esforços coletivos tem sido uma prática usual em problemas complexos. Especialmente nas pesquisas em saúde, métodos baseados em grandes comunidades têm se mostrado uma ferramenta robusta para superar desafios médicos, como mapear e reconstruir genes (MARBACH et al., 2012). No campo organizacional, a adoção de soluções a partir de grandes comunidades de usuários tem criado serviços, melhorado produtos e corrigido falhas com certa frequência (BRABHAM, 2013). Esta prática, quando extrapolada ou polarizada, afasta o conceito empoderador e participativo da cooperação em rede e transforma o indivíduo em um agente passivo para coleta e exploração de dados (FENG et al., 2019; VIANNA; MENEGHETTI, 2020). Quando organizada, a adoção de esforços coletivos em rede concebe e mantém com precisão, integridade e atualidade mais de seis milhões de artigos em uma enciclopédia (KITTUR; KRAUT, 2008). Sendo assim, as multidões de voluntários que se dispuseram a participar das iniciativas de civic hackathons (YUAN; GASCO-HERNANDEZ, 2019) somente conseguirão enfrentar os efeitos da pandemia do novo coronavírus com esforços adequadamente coordenados (BACQ et al., 2020).

A coordenação de esforços coletivos é um tema recorrente desde que Provan e Millward (1995) publicaram o artigo seminal sobre as redes de assistência à saúde nos Estados Unidos. A partir daí, duas grandes linhas de investigação se estabeleceram. A primeira delas tem ênfase na macrocoordenação das redes, enfocando os modos genéricos de governança e a sua eficácia em diferentes contextos (PROVAN; KENIS, 2008; EMERSON; NABATCHI; BALOGH, 2012). A segunda linha enfatiza a microcoordenação de redes, ou seja, as funções e os mecanismos que as fazem alcançar os objetivos almejados (ALBERS, 2010; MORETTI, 2017).

Ambas as linhas enriqueceram o conhecimento sobre a coordenação dos esforços coletivos e abriram janelas para novas investigações. No entanto, a ascensão do fenômeno da cooperação em grande escala na forma de hackathons levanta questionamentos sobre as possibilidades de adoção dos pressupostos e dos conceitos estabelecidos (LIFSHITZ-ASSAF; LEBOVITZ; ZALMANSON, 2020). Hackathons podem ajudar a enfrentar os desafios de uma crise pandêmica? Como os mecanismos de coordenação de rede se adequam à velocidade do processo e à dinâmica das decisões em hackathons? A interação, o 
envolvimento e o alinhamento dos participantes são afetados pelas particularidades dos hackathons? O perfil dos participantes e os papéis designados seguem sendo relevantes para o alcance dos resultados?

Ao buscar responder tais questões, este artigo se soma a um conjunto enorme de esforços de acadêmicos brasileiros dedicados a lidar com as consequências da Covid-19 (VENTURA et al., 2020). Alicerçado na microcoordenação de redes e nas evidências de uma das iniciativas, Hack for Brazil | Covid-19, o artigo objetiva compreender como funções e mecanismos de coordenação são adotados em contextos de cooperação em grande escala e intenso dinamismo. Além da contemporaneidade e de sua relevância social, este estudo se justifica por estabelecer uma robusta articulação teórico-empírica. Duas contribuições se destacam. Academicamente, o artigo avança no conhecimento sobre a microcoordenação de redes ao demonstrar como ela é empregada em iniciativas com milhares de envolvidos e pressão por resultados imediatos. Gerencialmente, o artigo contribui diretamente às iniciativas de apoio e suporte às crises graves, ao apontar funções e mecanismos que devem ser levados em consideração pelos proponentes para coordenar multidões de voluntários e alcançar os objetivos almejados.

O artigo está organizado em seis seções, incluindo esta introdução. Na próxima seção, são apresentadas e discutidas as categorias de análise da microcoordenação de redes. A terceira seção aborda a escolha do método de estudo de caso e os procedimentos metodológicos do estudo, tendo ênfase na coleta de evidências por meio de grupo focal. A quarta seção descreve o caso do Hack for Brazil | Covid-19 e as etapas e resultados de sua execução. A quinta seção utiliza as evidências coletadas junto aos envolvidos no caso para discutir as escolhas relativas à coordenação do hackathon. A última seção, além de destacar as principais contribuições e limitações do estudo, apresenta um conjunto de possibilidades de novos estudos que irão ajudar a entender e a aprimorar a coordenação de iniciativas similares.

\section{A MICROCOORDENAÇÃO DE REDES}

Em um estudo centenário, Galton (1907) evidenciou que um grande grupo de pessoas poderia reunir uma sabedoria coletiva superior a um indivíduo isolado. Oito décadas depois, esse poder dos grandes grupos foi retomado por pesquisadores sociais que observaram nas multidões uma riqueza de saberes que engloba tanto as individualidades quanto a sua totalidade (CANETTI, 1984; HARDT; NEGRI, 2005). Outra década se passou e as tecnologias de comunicação e de informação empoderaram as multidões de forma (c) (1) @ 2 REAd | Porto Alegre - Vol. 26 - N. ${ }^{\text {o }} 2$ - Maio / Agosto 2020 - p. 238-264. 
exponencial. Com a adoção de redes distribuídas, tornou-se tecnicamente viável a cooperação entre milhares de pessoas para resolver problemas complexos ou realizar tarefas desafiadoras (MICHELUCCI; DICKINSON, 2016; ROBINSON; JOHNSON, 2016). Mas, ainda que tecnicamente viável, as particularidades das multidões distribuídas (LUZ, 2016), como o número de participantes, o distanciamento social e a heterogeneidade de opiniões e interesses, proporcionam novos desafios para as práticas de cooperação em rede (BORTOLASO; VERSCHOORE, 2013).

Objetivos que demandam o esforço coletivo tendem a sofrer com um efeito deletério na qualidade dos resultados com o aumento de participantes (GLANCE; HUBERMAN, 1994). Apesar disso, em alguns domínios, a cooperação em grandes grupos se mostrou frutífera. Estudos apontam uma forte correlação entre o número de redatores e editores, a qualidade de um verbete na Wikipédia (WILKINSON; HUBERMAN, 2007) e a ampliação do aprendizado de graduandos quando há envolvimento em hackatons (KIENZLER; FONTANESI, 2017). Estudos brasileiros demonstram que hackathons promovem participação social, aumentam a transparência e geram inovações no setor público (FERREIRA; FARIAS, 2019). Para que isso aconteça, formas de microcoordenação explícitas e implícitas têm sido adotadas para aproveitar efetivamente a sabedoria da multidão em ambientes online (KITTUR; KRAUT, 2008). Essa microcoordenação de redes envolve questões que se complexificam à medida que os grupos crescem em tamanho.

Uma delas abrange a interação entre os participantes. A interação é a essência das iniciativas que promovem solução de problemas e a criação de valor de forma coletiva (RATTEN, 2020). A interação amplia o compartilhamento de informações e atividades e ajuda a alinhar decisões coletivas (SØRENSEN; TORFING, 2005). O conhecimento é mais provável de ser transferido quando há uma constante interação entre os participantes do que por troca de documentos ou consultas a bancos de dados (PAHOR; ŠKERLAVAJ; DIMOVSKI, 2008). Resolver problemas complexos em um curto espaço de tempo, como os hackatons se propõem, requer reflexão e profundidade de análise em um ambiente de negociação (AGRANOFF; MCGUIRE, 2001). Por isso, a microcoordenação de redes atua no sentido de ampliar as interações, bem como identificar e promover as interações produtivas e reduzir as improdutivas (CROSS et al., 2008). Ela abrange potencializar as capacidades já existentes e buscar as capacidades faltantes nos participantes menos integrados.

Outra questão enfrentada pela microcoordenação de redes é a do alinhamento de interesses. Usualmente, a diversidade de objetivos, escopos e abordagens se amplia à medida 
que o número de participantes de uma rede cresce (GLANCE; HUBERMAN, 1994). Esta é a riqueza das redes, que possibilita uma sabedoria coletiva superior à sabedoria individual, desde que alinhada por um senso de interesse comum (BACQ et al., 2020). O alinhamento diz respeito à demarcação dos limites de atuação da rede, ao direcionamento dos esforços coletivos e à conciliação de dissensos (ACAR; GUO; YANG, 2008; CRISTOFOLI; MACCIÒ; PEDRAZZI, 2015). Os estudos de Bodin (2017) e de Nohrstedt e Bodin (2019) demonstraram que a eficácia da colaboração em rede é determinada pelo alinhamento dos participantes com as possibilidades de ação coletiva. Como o alinhamento depende dos vínculos sociais e dos valores compartilhados (MARIANI, 2016; NOHRSTEDT; BODIN, 2019), a microcoordenação de redes irá requerer também um amplo envolvimento dos participantes.

O Envolvimento compreende a integração dos participantes nas ações e decisões da rede. A motivação de cada participante afeta seu nível de envolvimento na rede e, por conseguinte, o volume de contribuições para a criação de conhecimento (KIESLINGER; PATA; FABIAN, 2009). O envolvimento depende de fatores motivacionais extrínsecos, nos quais a recompensa é externa à atividade, e fatores motivacionais intrínsecos, nos quais a própria atividade é a recompensa (LIN, 2007). Estudos realizados em contextos online e hackathons apontam uma maior importância dada pelos participantes aos fatores intrínsecos, como aprendizagem, satisfação e reconhecimento (PINTO; SANTOS, 2018, FERREIRA; FARIAS, 2018). Assim, a microcoordenação de redes atua com o intuito de gerar relevância e valor intrínseco para atrair e envolver participantes (AMICHAI-HAMBURGER et al., 2016). Compete às iniciativas de cooperação em rede criar e implementar incentivos de envolvimento alinhados às diferentes motivações dos participantes (SAHIN; ROBINSON, 2002).

Diferentes níveis de envolvimento são explicados pelos diferentes níveis de motivação de cada participante (YASEEN; AL OMOUSH, 2020). Nesse sentido, outra questão relevante enfrentada pela microcoordenação de redes é o perfil dos participantes e sua homogeneidade ou heterogeneidade (CROSS et al., 2008). Perfis homogêneos facilitam a interação, mas dificultam a criatividade, tendo em vista que as contribuições são influenciadas por visões dominantes e pelas mesmas perspectivas. Perfis heterogêneos dificultam a interação, mas provocam a exposição e o choque de ideias que são necessários à inovação (PAHOR; ŠKERLAVAJ; DIMOVSKI, 2008). O perfil dos envolvidos afeta o tipo de contribuição (ARISTEIDOU; SCANLON; SHARPLES, 2017). Participantes ativos compartilham suas 
ideias e contribuições assiduamente, enquanto participantes passivos preferem navegar e aguardar um chamado específico para contribuírem (AMICHAI-HAMBURGER et al., 2016). Estudos indicam que ambos os perfis são necessários e complementam-se nos esforços coletivos (MALINEN, 2015). A microcoordenação de redes atua no intuito de identificar os perfis envolvidos na rede, para buscar um equilíbrio entre a intensidade e o tipo de contribuição.

Esse equilíbrio é possível por meio dos papéis exercidos pelos participantes na rede. O envolvimento diferenciado pode ser reconhecido por meio da atribuição de papéis no processo de geração, compartilhamento e escolhas de ideias. Uma rede requer várias funções de suporte para atender demandas específicas, como a promoção da participação, a moderação dos debates, ente outras (SCARSO; BOLISANI, 2008). A organização das atividades e processos que orientam os participantes da rede é facilitada quando papéis formais são atribuídos (CRISTOFOLI; MACCIÒ; PEDRAZZI, 2015). Os papéis nem sempre são decididos arbitrariamente, eles podem emergir na própria interação entre os participantes e nas rotinas geradas pelas relações sociais estabelecidas (MARIANI, 2016). Por exemplo, os participantes especialistas se destacam por suas contribuições dado o domínio de um tema, mas podem não ser os mais recomendados para estimular novas contribuições e conectar diferentes ideias. Papéis de moderadores e facilitadores, por sua vez, podem ser formalmente atribuídos e podem surgir informalmente na dinâmica interativa. A microcoordenação de redes, portanto, beneficia-se dessa dinâmica de papéis formais e informais para organizar atividades e orientar a participação dos envolvidos.

O esforço conjunto para mitigar os efeitos da pandemia do novo coronavírus, na forma da maratona online Hack for Brazil | Covid-19, demonstra as potencialidades da cooperação e conforma um campo empírico rico para o estudo da coordenação de redes. Ao elencar as questões da complexa microcoordenação da cooperação em redes de grande escala, este artigo se alinha aos estudos que enfatizam a eficácia de funções e mecanismos para o alcance dos objetivos coletivos (ALBERS, 2010; MORETTI, 2017). Deste referencial teórico, cinco categorias de análise foram destacadas: interação; alinhamento; envolvimento; perfil; e papéis. Eles estão sintetizados no Quadro 1 em orientação às discussões dos resultados para a microcoordenação de redes.

Quadro 1. Moldura de análise da microcoordenação de redes

\begin{tabular}{|l|c|c|}
\hline \multicolumn{1}{|c|}{ Categorias } & Descrição Sintética & Referências \\
\hline Interação & A interação é a essência da cooperação em & AGRANOFF; MCGUIRE, (2001); \\
\hline
\end{tabular}

(c) $\frac{(1) \Theta(8)}{\text { ar }}$ REAd | Porto Alegre - Vol. 26 - N. ${ }^{\circ} 2$ - Maio / Agosto 2020 - p. 238-264. 


\begin{tabular}{|c|c|c|}
\hline & $\begin{array}{l}\text { rede. A microcoordenação atua no sentido } \\
\text { de ampliar as interações, bem como } \\
\text { identificar e promover as interações } \\
\text { produtivas e reduzir as improdutivas. }\end{array}$ & $\begin{array}{l}\text { CROSS et al., (2008); } \\
\text { PAHOR; ŠKERLAVAJ; DIMOVSKI, (2008); } \\
\text { RATTEN (2020); } \\
\text { SØRENSEN; TORFING, (2005). }\end{array}$ \\
\hline Alinhamento & $\begin{array}{l}\text { O alinhamento diz respeito à demarcação } \\
\text { dos limites de atuação da rede, ao } \\
\text { direcionamento dos esforços coletivos e à } \\
\text { conciliação de dissensos. }\end{array}$ & $\begin{array}{l}\text { ACAR; GUO; YANG, (2008); } \\
\text { BACQ et al., (2020); } \\
\text { BODIN, (2019); } \\
\text { CRISTOFOLI; MACCIÒ; PEDRAZZI, (2015); } \\
\text { MARIANI, (2016); } \\
\text { NOHRSTEDT; BODIN, (2019). }\end{array}$ \\
\hline Envolvimento & $\begin{array}{l}\text { O envolvimento compreende a integração } \\
\text { dos participantes nas ações e decisões da } \\
\text { rede. A microcoordenação de redes atua } \\
\text { com o intuito de gerar relevância e valor } \\
\text { para atrair e envolver participantes. }\end{array}$ & $\begin{array}{l}\text { AMICHAI-HAMBURGER et al., (2016); } \\
\text { FERREIRA; FARIAS, (2018); } \\
\text { KIESLINGER; PATA; FABIAN, (2009); } \\
\text { LIN, (2007); } \\
\text { PINTO; SANTOS (2018); } \\
\text { SAHIN; ROBINSON, (2002); }\end{array}$ \\
\hline Perfil & $\begin{array}{l}\text { Perfis homogêneos ou heterogêneos } \\
\text { afetam o tipo de contribuição dos } \\
\text { participantes. A microcoordenação atua } \\
\text { no intuito de identificar os perfis } \\
\text { envolvidos para buscar um equilíbrio } \\
\text { entre a intensidade e o tipo de } \\
\text { contribuição. }\end{array}$ & $\begin{array}{l}\text { AMICHAI-HAMBURGER et al., (2016); } \\
\text { ARISTEIDOU; SCANLON; SHARPLES, (2017) } \\
\text { CROSS } \text { et al., (2008); } \\
\text { MALINEN, (2015); } \\
\text { PAHOR; ŠKERLAVAJ; DIMOVSKI, (2008) } \\
\text { YASEEN; AL OMOUSH, (2020). }\end{array}$ \\
\hline Papéis & $\begin{array}{l}\text { A microcoordenação de redes se beneficia } \\
\text { da dinâmica de papéis formais e informais } \\
\text { para organizar atividades e orientar a } \\
\text { participação dos envolvidos. }\end{array}$ & $\begin{array}{l}\text { CRISTOFOLI; MACCIÒ; PEDRAZZI, (2015); } \\
\text { MARIANI, (2016); } \\
\text { SCARSO; BOLISANI, (2008). }\end{array}$ \\
\hline
\end{tabular}

$\mathrm{Na}$ próxima seção, serão discutidas as escolhas metodológicas e detalhados os procedimentos que foram adotados para a coleta e a análise das evidências de campo.

\section{PROCEDIMENTOS METODOLÓGICOS}

Este artigo se insere no intenso processo de reflexão e de criação de conhecimento motivado pela pandemia do novo coronavírus. O estudo aborda o fenômeno das maratonas online, conhecidas como hackathons, pela ótica da microcoordenação de redes de grande escala. Para tanto, optou-se por realizar uma pesquisa qualitativa. A escolha se justifica pelas nuances e subjetividades do tema abordado, no qual os pressupostos teóricos estão em consolidação e as categorias de análise ainda não estão claramente definidas (CRESWELL, 1998). A riqueza e a atualidade da iniciativa Hack for Brazil | Covid-19 motivaram a adoção do método de estudo de caso. Tendo em vista que o objetivo do artigo abrange como as questões que afetam a microcoordenação de redes são adotadas em um contexto de grande escala e intenso dinamismo, o estudo de um caso fornece a profundidade de observação (c) (1) 9 REAd | Porto Alegre - Vol. 26 - N. ${ }^{\circ} 2$ - Maio / Agosto 2020 - p. 238-264. 
requerida para apresentar uma visão detalhada do fenômeno (DARKE; SHANKS; BROADBENT, 1998). Busca-se, neste artigo, uma generalização analítica dos resultados (YIN, 2010), confrontando as profusas evidências do caso Hack for Brazil | Covid-19 aos recentes avanços da microcoordenação de redes.

Optou-se por estudar os participantes do hackathon em seu próprio ambiente para evitar descobertas artificiais fora do contexto estabelecido (CRESWELL, 1998). As evidências foram coletadas a partir de fontes primárias e secundárias. As fontes secundárias se constituíram de documentos físicos publicados pelos organizadores do Hack for Brazil | Covid-19 e materiais digitais disponíveis nas plataformas utilizadas pelos participantes e em sites especializados. As fontes primárias foram obtidas por entrevistas exploratórias e, sobretudo, por meio de um grupo focal realizado com os envolvidos. As entrevistas foram realizadas com um dos idealizadores do hackathon, com uma das coordenadoras das atividades online e com uma participante voluntária. As três entrevistas tiveram uma ênfase exploratória e visaram a a) conhecer a iniciativa em toda a sua complexidade, b) discernir as perspectivas e percepções do fenômeno entre envolvidos e c) identificar um conjunto diversificado de participantes para compor o grupo focal. Em média, as entrevistas levaram 55 minutos de duração e auxiliaram a delimitar as questões relativas à microcoordenação de redes.

A escolha da coleta de evidências por grupo focal se deu pela interdependência dinâmica entre as percepções, opiniões e reações dos participantes que esta técnica propicia (COLLIS; HUSSEY, 2005). A dinâmica de pares em grupo estimula o compartilhamento das experiências e, sobretudo, provoca sentimentos e realça memórias que tornam as contribuições individuais dos participantes mais fiéis ao fenômeno (KRUEGER; CASEY, 2008). A coleta ocorreu no mês de maio de 2020, cerca de 40 dias após a realização do hackathon. Em decorrência da necessidade de isolamento social na data, optou-se pela realização do grupo focal na plataforma MS Teams ${ }^{\circledR}$, apoiando-se nas possibilidades de comunicação, colaboração, gravação e armazenamento da ferramenta. A seleção dos participantes teve como parâmetros o gênero, a procedência, a profissão, o papel desempenhado e a intensidade da atuação. A intensidade foi estabelecida de acordo com a contribuição de cada participante nas atividades do hackathon, parametrizada pelo seu protagonismo online e nos grupos de trabalho. Assim, a alta intensidade consistia na participação do respondente como protagonista nas atividades online ou na interação com o seu grupo de trabalho. A média intensidade consistia na participação sem protagonismo nas 
atividades online ou na interação com o seu grupo de trabalho. A baixa intensidade consistia na falta de participação nas atividades on-line ou na interação com o seu grupo de trabalho. Ao todo, seis indivíduos aceitaram participar do grupo focal em estudo. As características dos entrevistados (E1, E2, E3) e dos participantes do grupo focal (P1, P2, P3 ...) são sintetizadas no Quadro 2, de forma aleatória, a fim de preservar a identidade dos respondentes.

Quadro 2. Caracterização dos entrevistados e dos participantes do grupo focal

\begin{tabular}{|c|c|c|c|c|c|}
\hline $\begin{array}{c}\text { Respon- } \\
\text { dente }\end{array}$ & Gênero & Estado & Profissão & $\begin{array}{c}\text { Papel } \\
\text { Desempenhado }\end{array}$ & $\begin{array}{c}\text { Intensidade } \\
\text { da Atuação }\end{array}$ \\
\hline E1 & Fem. & RS & Administrador & Gestor geral & Alta \\
\hline E2 & Masc. & RS & Administrador & Gestor geral & Alta \\
\hline E3 & Fem. & RS & Arquiteto & Membro de GT & Média \\
\hline P1 & Masc. & AM & Empresário & Membro de GT & Média \\
\hline P2 & Masc. & RS & Empresário & Mentor de GT & Alta \\
\hline P3 & Masc. & MS & Engenheiro & Membro de GT & Média \\
\hline P4 & Fem. & RS & Publicitário & Líder de GT & Média \\
\hline P5 & Masc. & CE & Cientista de Dados & Líder de GT & Alta \\
\hline P6 & Masc. & RS & Engenheiro & Mentor de GT & Alta \\
\hline
\end{tabular}

A operacionalização do grupo focal seguiu um roteiro semiestruturado que fora validado junto aos entrevistados na etapa exploratória. No desenrolar de duas horas, travaramse debates relativamente informais sobre as questões que abarcam a microcoordenação de redes e, principalmente, sobre as provocações do pesquisador em relação aos construtos destacados na moldura de análise deste estudo. Para a interpretação dos resultados, os debates travados foram gravados e transcritos. Adotou-se a análise de conteúdo com a utilização dos construtos da microcoordenação de redes como categorias principais (BARDIN, 2011). O material coletado foi explorado tendo como orientação o sistema de codificação estabelecido no Quadro 2, visando à identificação, à repetição, à significação e à compreensão das cinco categorias do estudo. Submeteram-se as evidências transcritas a uma triangulação analítica com as fontes secundárias de informações e com os pressupostos do referencial teórico. Os resultados foram tratados em ciclos textuais e conceituais e analisados sob a lógica inferencial (BARDIN, 2011). Na próxima seção, será apresentada em detalhes a iniciativa Hack for Brazil | Covid-19 e, na seção seguinte, os resultados da microcoordenação de redes serão discutidos em profundidade. 


\section{APRESENTAÇÃO DO CASO}

O Hack for Brazil | Covid-19 foi uma iniciativa de hackathon online com o objetivo de buscar ideias estruturadas para combater os impactos da Covid-19 na sociedade. A iniciativa foi organizada pela aceleradora de startups Grow+ e executada por meio de uma parceria entre 40 instituições, que envolvia, entre elas, a Secretaria de Inovação, Ciência e Tecnologia do Rio Grande do Sul e a Prefeitura de Porto Alegre. Os organizadores tinham como meta receber cerca de 200 e 300 ideias que pudessem resultar em 20 soluções exequíveis para ajudar a sociedade brasileira a lidar com a crise do novo coronavírus. No lançamento do Hack for Brazil | Covid-19, o Secretário de Inovação, Ciência e Tecnologia comentou que a iniciativa era pautada pelas necessidades de saúde, com uma visão positiva de focar em problemas que estão sendo definidos por especialistas (JORNAL DO COMÉRCIO, 2020).

A realização de um hackathon online não foi a primeira escolha dos organizadores. Motivados por colegas portugueses que tinham feito um hackathon presencial com uma dezena de programadores para desenvolver soluções para a pandemia, os organizadores decidiram realizar algo semelhante no Brasil. A aceleradora trabalhava com inovação aberta e colaborativa e tinha o conhecimento e os contatos requeridos para propor uma iniciativa de hackathon para ajudar a lidar com o novo coronavírus. Como já haviam feito hackathons presenciais, ponderaram que, se realizassem uma iniciativa online, poderiam atingir mais pessoas. Conforme o relato de um dos entrevistados, "Vamos fazer o seguinte: um hackathon online que podemos ter 200 pessoas. A gente construiu o hackathon pensando em ter 200 pessoas, seria uma das maiores maratonas de inovação aberta do Brasil, uma coisa maluca" (Entrevistado E2).

Como não teriam estrutura suficiente para realizar uma iniciativa de tamanho porte, os organizadores logo se preocuparam em buscar parceiros que a viabilizassem. Fizeram uma chamada aberta para as empresas e instituições com as quais se relacionavam. "Estendemos o convite de forma aberta aos parceiros e as pessoas foram ingressando nos links que nós disponibilizamos, de acordo com determinados perfis ou temáticas: tecnologia, comunicação e divulgação, investimentos" (Entrevistado E1). Em uma semana, chegaram a 40 empresas parceiras, além de secretarias do estado e do município. Como receberam apoio de empresas de fora do Rio Grande do Sul, decidiram tornar o hackathon nacional e, assim, surgiu o nome Hack for Brazil | Covid-19. 
A rapidez no processo de formação de parcerias dificultou a coordenação entre os envolvidos. Conforme os entrevistados, alguns parceiros não se engajaram completamente, outros não compartilharam todas as informações e recursos que possuíam, outros desconheciam o funcionamento de um hackathon e não sabiam como ajudar. Havia divergências de visões. Alguns parceiros defendiam que o hackathon abrangesse somente startups e outros acreditavam que a iniciativa não teria condições para ir além do Rio Grande do Sul. Apesar das diferenças, o aumento do grupo de organizadores para 40 empresas e instituições possibilitou estruturar o hackathon de forma instantânea. Em dois dias, foi desenvolvida e disponibilizada a plataforma de operacionalização do hackathon. Na mesma semana, os envolvidos abriram a plataforma para a inscrição de participantes. A repercussão foi maior do que os 200 ou 300 participantes inicialmente aguardados. "Em uma quinta-feira, estávamos com 500 registros, depois foi para 750, depois foi para 1.500, e quando chegou em 2.000, a gente pensou: não acredito, como vamos fazer isso agora? Ficamos com mais de 2.300 inscritos" (Entrevistado E2).

A maratona ocorreu entre os dias 23 e 27 de março de 2020. Ela foi estruturada em cinco etapas. A primeira etapa consistia na distribuição dos inscritos em grupos de trabalho. A etapa seguinte compreendia o conhecimento dos futuros usuários das soluções, denominados de personas, como os idosos, as pessoas em quarentena, os assintomáticos, entre outros. A terceira etapa abrangia o detalhamento da realidade de cada persona pelos grupos de trabalho para mapear oportunidades, sendo denominada de jornada do usuário. A quarta etapa, chamada de ideação, compreendia a identificação, a discussão e a apresentação das ideias de soluções por cada grupo de trabalho. Por fim, a quinta etapa finalizava o hackathon com a seleção de 20 ideias para desenvolvimento. Essa etapa ficou ao encargo de uma banca de especialistas convidados pela organizadora e pelos parceiros da iniciativa.

De acordo com o relatório elaborado para angariar fundos às soluções concebidas, o Hack for Brazil | Covid-19 teve 2.350 inscritos e mais de 1.000 participantes engajados. Os participantes tinham a idade média de 32 anos e se concentravam nas regiões Sul (506) e Sudeste (271). Foram formados 50 grupos de trabalho multidisciplinares, os quais geraram mais de 500 ideias contemplando as temáticas de conscientização e prevenção, eficiência operacional, diagnóstico e tratamento, entre outras. As ideias propostas envolviam tecnologias como redes neurais, inteligência artificial, biotecnologia, impressão 3D e internet das coisas. Em decorrência do grande número de participantes, os especialistas convidados optaram por selecionar 50 e não 20 ideias para desenvolvimento. Foi realizado um processo de análise por 
passos, no qual os especialistas categorizavam e avaliavam ideia por ideia. Conforme evidencia um dos entrevistados, "A gente construiu times de três a cinco pessoas avaliando cada um dos grupos, cada uma das ideias dos grupos e aí nós chegamos a 184 ideias. Categorizamos essas ideias e nós fomos a 50 ideias" (Entrevistado E2).

Segundo o relatório obtido, as ideias selecionadas atendem, principalmente, a população assintomática (37\%), os serviços de saúde (25\%) e os profissionais da saúde (22\%). Algumas das ideias estão em operação e inclusive em processo de ampliação da abrangência de atuação, como o exemplo dos arquitetos voluntários mencionado na introdução. De acordo com um dos participantes, “A gente está com nove hospitais e estamos em contato com mais dois hospitais, mas o objetivo realmente do projeto é alcançar as UPAs e, após isso, os postos de saúde, porque temos que chegar na situação mais crítica, que é a raiz do problema da saúde" (Entrevistado E3).

Outras ideias estão em um estágio anterior, distantes da sua utilização. Elas são categorizadas em ideias em descoberta e ideias em construção. As primeiras são aquelas que nasceram no próprio hackathon e precisam ser estruturadas pelos participantes. As ideias em construção estão mais avançadas. Já foram estruturadas pelos grupos de trabalho e se encontram no processo de validação. Os organizadores estão trabalhando para desenvolver cada uma das 50 ideias. Há um tipo de abordagem para cada estágio, desde a estruturação das ideias, até a elaboração de pilotos para demonstração. Além disso, os participantes seguem aprimorando as ideias geradas no Hack for Brazil | Covid-19. Nas palavras de um participante do grupo focal: "Nosso grupo está bem focado para manter vivas todas as ideias para adiante. Nossas ideias são negócios sociais que não visam necessariamente o lucro. Então, para nós, tem sido um grande desafio criar isso" (Participante P3).

Os próximos passos abrangem a apresentação das ideias para startups e comunidades de desenvolvedores, a busca de recursos junto a investidores e a empresas apoiadoras e, ao fim, a disponibilização das soluções para os usuários e demais partes interessadas. Há uma expectativa de que as ideias geradas sejam desenvolvidas e atinjam o público-alvo, embora os passos seguintes ainda estejam em um processo de construção. De acordo com um dos participantes: "A gente cria uma expectativa muito grande em relação aos próximos passos e, de novo, a gente está construindo-os à medida que eles vão acontecendo (...) A gente está prospectando investidores, está buscando empresas, está buscando editais, está buscando conexões com as grandes entidades e com os futuros patrocinadores e investidores para cada uma das ideias" (Participante P6). 
Independentemente do avanço das ideias, o sentimento comum entre os participantes do Hack for Brazil | Covid-19 foi de satisfação e contentamento com a oportunidade de contribuir para o enfrentamento do novo coronavírus. "Foi um movimento humano muito bonito (...) Estou muito feliz. Não é só o lado ruim. A gente não está só sofrendo, a gente tá podendo mostrar que é capaz de enfrentar e de superar" (Participante P4). Um dos participantes reforçou a sensação de empoderamento decorrente da oportunidade de propor e gerar soluções conjuntas. "Me tocou viver isso. Nos tirou da passividade para ter uma sensação de domínio, de usar a nossa criatividade para contribuir. Esse hackathon foi uma oportunidade humana de estar fazendo parte disso. Uma sensação de empoderamento diante de algo que sobrepassa a todos" (Participante P1). Os participantes também destacaram, em consenso, o esforço empreendido para viabilizar a iniciativa do hackathon e os desafios de coordenação enfrentados durante a realização das atividades. Estas questões e especialmente a microcoordenação de redes serão discutidas na próxima seção.

\section{DISCUSSÃO DOS RESULTADOS}

O crescimento de iniciativas de cooperação em grande escala, como o Hack for Brazil | Covid-19, trouxe novos questionamentos para as investigações sobre a coordenação dos esforços coletivos. Entre eles, destacam-se os efeitos da velocidade do processo e do dinamismo do contexto nas decisões dos envolvidos (BACQ et al., 2020). As entrevistas e o grupo focal evidenciaram esses efeitos e demonstraram sua implicação nas decisões de coordenação. Os entrevistados, em sintonia, ressaltaram que a velocidade do processo afetou negativamente o intuito inicial de se ter uma autogestão e uma divisão da microcoordenação entre os parceiros. "O objetivo era que nós tivéssemos coordenadores em cada frente, para que a coordenação não ficasse conosco, tão somente" (Entrevistado E1). Entretanto, nem todos os parceiros dominavam o funcionamento de um hackathon ou tinham recursos humanos para se engajar na coordenação. "As entidades que foram entrando no projeto iam criando uma expectativa que iriam ajudar na gestão do projeto. Mas só ficavam olhando, fazendo mentoria e não ajudavam tanto" (Participante P6).

Assim, gradualmente, a microcoordenação do Hack for Brazil | Covid-19 foi sendo assumida pela aceleradora que originalmente propôs a iniciativa. A ascensão de uma organização líder, quando a autogestão perde eficiência, não é um movimento novo. Trata-se de um fenômeno encontrado e debatido na literatura de cooperação em rede desde os anos 
1990 (GRANDORI; SODA, 1995; DUSSAUGE; GARRETTE, 2000). A peculiaridade no fenômeno do hackathon é a velocidade na qual a tensão entre a eficiência e a inclusão se estabelece e afeta as escolhas de coordenação (PROVAN; KENIS, 2008). Em menos de duas semanas, a rede de parceiros foi formada, as decisões foram inicialmente compartilhadas, as dificuldades de engajamento surgiram e a coordenação por meio de uma organização líder foi assumida como o modo mais pertinente de condução da iniciativa.

Um primeiro achado, portanto, é evidenciar que o dinamismo e a velocidade do processo de operação, característicos dos hackathons, afetam as decisões de microcoordenação da cooperação em rede (BORTOLASO; VERSCHOORE, 2013). Para que as atividades ocorressem a contento em poucos dias, a organização líder optou por definir, previamente, os 50 grupos de trabalho de acordo com o perfil dos participantes e distribuir incumbências aos indivíduos conforme o papel que lhes eram atribuídos. O entrevistado E2 explicou esta decisão: “A formação dos GTs partia do pressuposto que deveriam ser multidisciplinares (...). Nós alocamos pessoas com diferentes perfis (...). Cada grupo deveria ter um médico, um designer, um desenvolvedor, um pesquisador, um estudante".

Esta decisão se vincula ao pressuposto teórico de que uma maior heterogeneidade dos perfis provoca um contraste de visões necessário à geração de inovação (CROSS et al., 2008). As mais de 500 ideias geradas indicam coerência com achados de estudos anteriores (PAHOR; ŠKERLAVAJ; DIMOVSKI, 2008). Os resultados encontrados no Hack for Brazil | Covid-19 também reforçam os postulados teóricos de que o perfil dos envolvidos afeta a intensidade das contribuições (ARISTEIDOU; SCANLON; SHARPLES, 2017). "Como os grupos foram randomicamente organizados, ninguém era do mesmo lugar, isso fez diferença na dinâmica, no processo, na inovação e até na percepção das pessoas do que fazer e como" (Participante P4). Além disso, os resultados empíricos demonstraram que os perfis ativos compartilhavam suas ideias assiduamente, ao passo que os perfis passivos preferiam aguardar um chamado para contribuir (AMICHAI-HAMBURGER et al., 2016). "Eu fui no grupo privado, peguei o celular de um a um, e perguntei, olha a gente está avançando, vocês querem participar? É muito importante que todo mundo esteja junto nessa" (Participante P4).

Por outro lado, a decisão da organização líder de buscar a heterogeneidade dos grupos de trabalho teve impacto negativo na interação entre os envolvidos. Nas palavras de um dos participantes, "Teve uma dificuldade muito grande de conseguir conectar as pessoas, porque o público era muito heterogêneo" (Participante P2). Estudiosos da cooperação em rede afirmam que a interação é essencial para difundir informações e compartilhar o conhecimento coletivo 
(SØRENSEN; TORFING, 2005; PAHOR; ŠKERLAVAJ; DIMOVSKI, 2008). No entanto, os resultados demonstraram que, em iniciativas de grande porte, como os hackathons, a interação ocorre apenas entre uma pequena parte dos envolvidos (RATTEN, 2020). Nas palavras de um dos participantes: "A interação no nosso grupo foi bem escassa. Tinha muita gente, eu não cheguei contar quantos tinham, mas devia ter, mais ou menos, uns 20. Mas eu acho que quem participou mesmo foi eu, a Ana e mais umas três ou quatro pessoas" (Participante P1).

Trata-se de um achado que se destaca na cooperação em redes online com milhares de envolvidos. A distância espacial e a velocidade de decisão modificam as formas antigas de interação e reestruturam as relações sociais existentes (VERSCHOORE, 2000). Como afirma um dos participantes, a interação nesse contexto tem a suas particularidades: "Não só um grupo bastante heterogêneo de características e de perfis, mas também um número muito grande de pessoas participantes do hackathon, que cria uma complexidade para gestão dos grupos e das ideias" (Participante P6). O tamanho e o dinamismo do fenômeno impossibilitam que uma organização líder coordene tantas interações simultâneas e muito menos que promova interações produtivas e minimize as improdutivas (CROSS et al., 2008). As decisões e ações não encontram um ambiente de negociação que possibilite reflexão e profundidade de análise (AGRANOFF; MCGUIRE, 2001) e acabam sendo executadas ad hoc e de forma orgânica nos grupos de trabalho, como afirma o Participante P5, "Eu tomei inciativa à frente. Me tornei mais proativo e observei o comportamento de cada pessoa para ver quem estava realmente comprometido e quem não estava. A partir daí, se chegava ao entendimento do coletivo".

A interação não teria efeito sem o envolvimento dos participantes e sem o alinhamento dos objetivos. A pressão temporal e a dinâmica de decisões dos hackathons repercutiram no envolvimento e no alinhamento dos envolvidos (LIFSHITZ-ASSAF; LEBOVITZ; ZALMANSON, 2020). Os participantes do grupo focal observaram, em consenso, a necessidade de tempo para que os indivíduos se sintam confortáveis em contribuir. Nas palavras de um participante: "Os grupos se formaram naquele momento. Tem hackathons que você pode até já ir com os seus colegas. Você já acaba meio que com uma pré-formação anterior. No online não teve isso. No início, no meu grupo específico, foi um pouco tímido no começo" (Participante P3). Coube aos próprios membros dos grupos de trabalho, de forma adaptativa, assumirem a função de envolvimento. "Eu tentei resgatar as pessoas que foram se perdendo, no momento em que a gente tinha que entregar alguma coisa" (Participante P4). Os 
resultados deste estudo apontam, portanto, que as entregas rotineiras solicitadas pela organização do hackathon se transformaram em um objeto de valor relevante para atrair e envolver os participantes (AMICHAI-HAMBURGER et al., 2016). Complementa-se assim, os achados de estudos brasileiros que indicam a importância dos fatores motivacionais intrínsecos (PINTO; SANTOS, 2018, FERREIRA; FARIAS, 2018), ao se acrescentarem as entregas rotineiras parciais como forma de satisfação e reconhecimento ao envolvimento.

Essas entregas se constituíram também no principal instrumento de microcoordenação de redes utilizado pela organização líder para alinhar os diversos interesses. Havia metas e um cronograma de entregas para os grupos de trabalho, que demarcavam os limites de atuação e direcionavam os esforços de criação e aperfeiçoamento das ideias (ACAR; GUO; YANG, 2008; CRISTOFOLI; MACCIÒ; PEDRAZZI, 2015). “O foco era baseado em metas. Isso, naturalmente, forçou as pessoas que tinham interesse a continuar e, as pessoas que não tinham interesse a desistir" (Participante P5). A velocidade e a dinâmica dos hackathons online não proporcionam o adequado fortalecimento de vínculos sociais ou de valores compartilhados (MARIANI, 2016; NOHRSTEDT; BODIN, 2019). O alinhamento dos interesses ocorre de forma mais intensa no calor das decisões, à medida que os prazos para as entregas se esgotam e a necessidade de se estabelecer um senso de interesse comum aflora (BACQ et al., 2020). "Ou estava junto e participava dentro do cronograma, ou seria equivalente à metáfora de um barco que saiu no mar e alguns outros pegaram um barquinho e colocaram no mar depois. A seleção natural fez com que o grupo tivesse uma linha de ação e aí, naturalmente, consolidou, fechou" (Participante P5).

A dinâmica do fenômeno dos hackathons online afeta, de forma similar, a microcoordenação de redes no que tange aos papéis formais estabelecidos pela organização líder (SCARSO; BOLISANI, 2008; CRISTOFOLI; MACCIÒ; PEDRAZZI, 2015). Este achado foi evidenciado pelo fato de os mentores definidos pela organização do Hack for Brazil | Covid-19 não terem tido a eficácia esperada em dirimir dúvidas e reduzir as assimetrias entre os grupos de trabalho. A intensidade e a velocidade das demandas prejudicaram o apoio dos mentores: "Não ficava sinalizado para o mentor a solicitação que já havia sido respondida e qual estava em aberto. A gente, como mentor, entrava na solicitação e só depois descobria que ela já tinha sido respondida" (Participante P6).

No contexto dos hackathons, os papéis que emergem da própria interação entre os participantes demonstram-se mais eficazes (MARIANI, 2016). Um dos participantes, que tinha o papel formal de mentor, ao se envolver intensamente com uma ideia, assumiu o papel 
de líder, ajudando e orientando o grupo de trabalho a alcançar o objetivo com seu conhecimento e ação: "Eu acabei me envolvendo porque gerei uma ideia. E acabei ajudando todo mundo. Eu tive que trabalhar como líder e consegui fazer com que a galera fizesse as entregas (....) como o grupo era bem heterogêneo tive que ajudar no be a bá" (Participante P2). Trata-se de um achado que reforça a característica adaptativa da coordenação de hackathons executados sob uma intensa pressão de chegar a resultados em pouquíssimo tempo (BACQ et al., 2020; LIFSHITZ-ASSAF; LEBOVITZ; ZALMANSON, 2020).

Por fim, os entrevistados e participantes do grupo focal destacaram a importância de a microcoordenação abarcar normas e regulamentos formais. Nas palavras de um entrevistado, "Um regulamento detalhado facilitaria a tomada de decisão. Saber qual vai ser o final do processo, o que eles vão ter que entregar, de que forma eles vão ter que entregar" (Entrevistado E1). Embora não englobados no framework desta pesquisa, estudos anteriores já haviam analisado a adoção de tais instrumentos no contexto da governança de redes (THERBORN, 2002). Normas e regulamentos formais explicitam aos envolvidos o que é esperado deles e como eles devem se comportar (PADILHA; VERSCHOORE, 2013). No contexto de hackathons, normas e regulamentos formais se mostram ainda mais relevantes devido à velocidade das ações: "É tudo muito rápido em um hackathon (...) e não ficou definido no início quais eram as etapas e as datas de entregas" (Participante P2).

Ainda que hackathons tenham uma coordenação adaptativa e as interações ocorram de forma dinâmica (LIFSHITZ-ASSAF; LEBOVITZ; ZALMANSON, 2020), os resultados aqui encontrados apontam que a explicitação do que se espera alcançar e, sobretudo, de como se espera alcançar, reforça a assertividade dos envolvidos e facilita o alcance dos objetivos coletivos (ALBERS, 2010; MORETTI, 2017). Nas palavras de um dos participantes do grupo focal: "Uma coisa que eu senti falta de início foi de um edital. Eu acho que seria importante pra gente ter tido esse documento a princípio, para ter uma ideia de onde a gente está, o que a gente está fazendo, quais são as regras, como as interações funcionam” (Participante P1).

Os resultados desta pesquisa reforçaram alguns dos postulados teóricos construídos na trajetória de estudos sobre a microcoordenação de rede e evidenciaram as principais diferenças existentes no contexto veloz e dinâmico das iniciativas online de grandes proporções. Não obstante, trazem complementações aos estudos recentes sobre a microcoordenação e sobre hackathons no contexto nacional e internacional. Os resultados são sintetizados no quadro 3, acompanhados das evidências coletadas no grupo focal. 
Quadro 3. Síntese dos resultados da pesquisa

\begin{tabular}{|c|c|c|}
\hline Categorias & Resultados & Evidências \\
\hline Interação & $\begin{array}{l}\text { Em iniciativas de grande porte, como } \\
\text { os hackathons online, a interação } \\
\text { ocorre apenas entre uma pequena parte } \\
\text { dos envolvidos. } \\
\text { O dinamismo impossibilita que uma } \\
\text { organização líder coordene tantas } \\
\text { interações simultâneas. A promoção } \\
\text { das interações produtivas ocorre de } \\
\text { forma orgânica nos grupos. }\end{array}$ & $\begin{array}{l}\text { "A interação no nosso grupo foi bem escassa. } \\
\text { Tinha muita gente, eu não cheguei contar quantos } \\
\text { tinham (...) Mas eu acho que quem participou } \\
\text { mesmo foi eu, a Ana e mais umas } 3 \text { ou } 4 \text { pessoas" } \\
\text { (Participante P1). } \\
\text { "Eu tomei inciativa à frente. Me tornei mais } \\
\text { proativo e observei o comportamento de cada } \\
\text { pessoa para ver quem estava realmente } \\
\text { comprometido" (Participante P5). }\end{array}$ \\
\hline Alinhamento & $\begin{array}{l}\text { No contexto dos hackathons, a pressão } \\
\text { temporal não proporciona o } \\
\text { fortalecimento de vínculos sociais ou } \\
\text { de valores compartilhados. } \\
\text { O alinhamento dos interesses ocorre de } \\
\text { forma mais intensa, no calor das } \\
\text { decisões, à medida que os prazos para } \\
\text { as entregas se esgotam. }\end{array}$ & $\begin{array}{l}\text { "Ou estava junto e participava dentro do } \\
\text { cronograma, ou seria equivalente à metáfora de } \\
\text { um barco que saiu no mar e alguns outros } \\
\text { pegaram um barquinho e colocaram no mar } \\
\text { depois. A seleção natural fez com que o grupo } \\
\text { tivesse uma linha de ação e aí, naturalmente, } \\
\text { consolidou, fechou” (Participante P5). }\end{array}$ \\
\hline Envolvimento & $\begin{array}{l}\text { A velocidade e a dinâmica dos } \\
\text { hackathons online dificultam que os } \\
\text { participantes se sintam confortáveis em } \\
\text { se envolver desde o início. } \\
\text { As entregas rotineiras solicitadas são } \\
\text { adotadas pelos envolvidos como um } \\
\text { objeto de valor relevante para atrair e } \\
\text { envolver os participantes. }\end{array}$ & $\begin{array}{l}\text { "No início, no meu grupo específico, foi um } \\
\text { pouco tímido no começo" (Participante P3). } \\
\text { "Eu tentei resgatar as pessoas que foram se } \\
\text { perdendo, no momento em que a gente tinha que } \\
\text { entregar alguma coisa" (Participante P4). }\end{array}$ \\
\hline Perfil & $\begin{array}{l}\text { Hackathons de grandes proporções } \\
\text { possibilitam reunir participantes de } \\
\text { perfis heterogêneos para ampliar a } \\
\text { geração de ideias. O equilíbrio entre a } \\
\text { intensidade e a contribuição é feito } \\
\text { organicamente pelos participantes. }\end{array}$ & $\begin{array}{l}\text { "Como os grupos foram randomicamente } \\
\text { organizados, ninguém era do mesmo lugar, isso } \\
\text { fez diferença na dinâmica, no processo, na } \\
\text { inovação e até na percepção das pessoas do que } \\
\text { fazer e como" (Participante P4). }\end{array}$ \\
\hline Papéis & $\begin{array}{l}\text { No contexto dos hackathons, os papéis } \\
\text { que emergem da própria interação } \\
\text { entre os participantes se mostram mais } \\
\text { eficazes do que os papéis estabelecidos } \\
\text { formalmente pela organização líder. }\end{array}$ & $\begin{array}{l}\text { "Eu acabei me envolvendo porque gerei uma } \\
\text { ideia. E acabei ajudando todo mundo. Eu tive que } \\
\text { trabalhar como líder e consegui fazer com que a } \\
\text { galera fizesse as entregas (....) como o grupo era } \\
\text { bem heterogêneo tive que ajudar no be a bá" } \\
\text { (Participante P2). }\end{array}$ \\
\hline Normas & $\begin{array}{l}\text { Em hackathons online de grandes } \\
\text { proporções, a clara explicitação do } \\
\text { comportamento esperado e do que } \\
\text { deve ser entregue ao final reforça a } \\
\text { assertividade dos envolvidos e facilita } \\
\text { o alcance dos objetivos coletivos. }\end{array}$ & $\begin{array}{l}\text { "Uma coisa que eu senti falta de início foi de um } \\
\text { edital. Eu acho que seria importante pra gente ter } \\
\text { tido esse documento a princípio, para ter uma } \\
\text { ideia de onde a gente está, o que a gente está } \\
\text { fazendo, quais são as regras, como as interações } \\
\text { funcionam" (Participante P1). }\end{array}$ \\
\hline
\end{tabular}

O quadro 3 teve como base as cinco categorias de análise organizadas a partir do referencial teórico: interação; alinhamento; envolvimento; perfil; e papéis. Além dos 
resultados para cada uma das categorias, o quadro é complementado pela inclusão da sexta categoria, que emergiu do campo empírico e foi denominada de "normas". Na próxima seção, serão destacadas as contribuições do estudo, seguidas das limitações decorrentes das escolhas de pesquisa e das oportunidades para trabalhos futuros.

\section{CONSIDERAÇÕES FINAIS}

A maratona online Hack for Brazil | Covid-19 se uniu ao crescente esforço mundial para mitigar os efeitos deletérios da pandemia do novo coronavírus. Alicerçado na sabedoria das multidões, o Hack for Brazil | Covid-19 fez surgir 50 ideias que passaram a ser desenvolvidas pelos participantes em conjunto com startups, empresas, investidores e o poder público. A iniciativa desse hackathon, desde sua origem até o posterior aprimoramento das ideias geradas, manifesta a potencialidade da ação coletiva e evidencia a necessidade do aprofundamento da compreensão sobre os diferentes aspectos da coordenação de redes. Este estudo teve como objetivo compreender como funções e mecanismos de coordenação são adotados em contextos de grande escala e intenso dinamismo, como o Hack for Brazil | Covid-19. Ele se alinha às recentes pesquisas que abordam o fenômeno no campo da administração pública e de empresas (FERREIRA; FARIAS, 2018; YUAN; GASCOHERNANDEZ, 2019; BACQ et al., 2020; LIFSHITZ-ASSAF; LEBOVITZ; ZALMANSON, 2020).

Os resultados do estudo assinalam contribuições acadêmicas e gerenciais. Em primeiro lugar, as evidências demonstraram que a cooperação em rede pode ajudar a enfrentar os desafios de uma crise pandêmica. Em apenas uma iniciativa, o Hack for Brazil | Covid-19, esforços coletivos geraram mais de 500 ideias para ajudar a conscientizar e prevenir a disseminação do vírus, diagnosticar a doença e melhorar a eficiência dos tratamentos com a utilização de redes neurais, inteligência artificial, biotecnologia e impressão 3D. Mais do que isso, as evidências destacaram o sentimento de empoderamento demostrado pelos envolvidos ao criarem soluções para enfrentar o novo coronavírus que não seriam possíveis se atuassem de maneira isolada.

Academicamente, o estudo trouxe contribuições para a microcoordenação de redes de grande escala em contextos dinâmicos e sob intensa pressão. A primeira delas é que as funções e mecanismos de coordenação são afetados pela dinâmica e pela velocidade das decisões nos hackathons. A interação se limita apenas a parte dos envolvidos e a necessária 
promoção das interações produtivas ocorre mais pela relação entre os participantes do que pela ação de uma liderança instituída. A rapidez dos processos de decisão dificulta o fortalecimento de vínculos sociais, afetando negativamente o envolvimento e o alinhamento entre os participantes. Nesse contexto, os resultados do estudo demonstraram que o alinhamento dos interesses ocorre de forma mais intensa no calor das decisões, à medida que os prazos para as entregas se esgotam.

Não obstante, o perfil dos participantes e os papéis designados afetam as funções e os mecanismos de coordenação de rede e seguem sendo relevantes para o alcance dos resultados. Os papéis estabelecidos formalmente pela liderança instituída são menos relevantes do que os papéis que surgem no decorrer das intensas atividades realizadas para tentar atender as entregas solicitadas. O preenchimento dos diversos papéis da microcoordenação das redes é facilitado pela ampla diversidade de perfis que hackathons com mais de mil participantes possibilitam. A heterogeneidade dos perfis enriquece as fontes para a origem de ideias e soluções, ao mesmo tempo que cria as condições para o necessário equilíbrio entre a intensidade do envolvimento e a riqueza de cada contribuição. Por último, o estudo evidenciou a relevância de normas e regulamentos formais para a microcoordenação de redes dinâmicas e de grandes proporções como hackathons. Os participantes podem se sentir inseguros sem uma clara explicitação dos objetivos, das entregas e dos comportamentos esperados.

O estudo também trouxe contribuições práticas para os promotores de iniciativas de cooperação similares. Os resultados demonstraram que a formação de grupos heterogêneos é um ponto crucial para o êxito de hackathons. Além disso, apontaram que as iniciativas terão uma maior assertividade dos envolvidos se, desde o início, ficar claro e bem definido o que se espera deles nas atividades-meio e nas entregas finais. Ainda assim, dúvidas e questionamentos surgirão. Para tanto, as evidências coletadas indicam a utilização de ferramentas de comunicação, como seminários online, chamados de webinars. Essas ferramentas possibilitam criar diferentes momentos de contato com os participantes, evitando que se sintam desassistidos, resolvendo dúvidas e questões e proporcionando o necessário envolvimento.

As contribuições acadêmicas e práticas mencionadas estão condicionadas às limitações deste estudo. Como apontado na seção de procedimentos metodológicos, o tema da microcoordenação de redes e o recente fenômeno dos hackathons possuem nuances que repercutem nas escolhas de pesquisa. Suas categorias de análise, por exemplo, ainda não estão 
claramente definidas. Assim, os resultados permitem uma generalização analítica do caso Hack for Brazil | Covid-19 e não devem ser ampliados para todas as iniciativas de hackathons. As evidências coletadas de fontes primárias ocorreram por meio de entrevistas e de grupo focal realizados em ambiente virtual tendo em vista o requerido isolamento social. Ainda que as tecnologias disponíveis permitam a visualização dos respondentes, não foi possível se obter a mesma riqueza interativa proporcionada pelas coletas presenciais.

Os resultados obtidos levantam novos questionamentos sobre a coordenação de redes. De que forma as funções e mecanismos da microcoordenação de redes são afetados em iniciativas de hackathons presenciais? Neste caso, permanecem as características da pressão temporal e da velocidade dos processos, mas com um número menor de participantes e uma maior homogeneidade entre eles. No mesmo contexto, questionam-se quais categorias são mais afetadas por esta presencialidade. Paralelamente, levantam-se tais questões para as iniciativas de hackathons de empresas, nas quais os esforços coletivos são delimitados pelos objetivos organizacionais. Essas questões abrem janelas de possibilidades para que novas pesquisas continuem aprofundando o conhecimento sobre a microcoordenação de redes.

Outras duas oportunidades de estudos futuros se relacionam à coordenação dos esforços coletivos em contextos dinâmicos. A pesquisa de Lifshitz-Assaf, Lebovitz e Zalmanson (2020) apontou o papel crítico da ambiguidade temporal para o sucesso das iniciativas de hackathons. Há, dessa forma, um caminho aberto para investigações que abordem a microcoordenação de redes de forma adaptativa aos processos acelerados de inovação. Esta requerida adaptação traz implicações aos trade-offs da coordenação de redes propostos por Provan e Kenis (2008). Novos estudos têm a oportunidade de investigar, portanto, como as tensões de coordenação de eficiência versus inclusão, legitimidade interna versus legitimidade externa, e flexibilidade versus estabilidade se comportam em contextos dinâmicos, intensos e de rápida tomada de decisão como os hackathons.

Crises de grandes proporções, como a motivada pela pandemia do novo coronavírus, bem como os problemas sociais de alta complexidade não são passíveis de enfrentamentos solitários por organizações ou governos. Eles requerem esforços coletivos coordenados. O conhecimento sobre a microcoordenação de redes tem crescido no campo organizacional e pode contribuir com a mitigação de tais questões. Espera-se que este estudo possa estimular outras tantas iniciativas de hackathons cívicos, como o Hack for Brazil | Covid-19, e instigar pesquisadores a se debruçarem sobre as questões da coordenação de redes, proporcionando 
avanços ao conhecimento e às atuais práticas empregadas pelos promotores. A sociedade irá agradecer.

\section{REFERÊNCIAS}

ACAR, M.; GUO, C.; YANG, J. Accountability when hierarchical authority is absent: views from public-private partnership practitioners. American Review of Public Administration, v. 38, n. 1, p. 3-23, 2008.

AGRANOFF, R.; MCGUIRE, M. Big questions in public network management research. Journal of Public Administration Research and Theory, v. 11, n. 3, p. 295-326, 2001.

ALBERS, S. Configurations of alliance governance systems. Schmalenbach Business Review, v. 62, n. 3, p. 204-233, 2010.

AMICHAI-HAMBURGER, Y.; GAZIT, T.; BAR-ILAN, J.; PEREZ, O.; AHARONY, N.; BRONSTEIN, J.; DYNE, T.S. Psychological factors behind the lack of participation in online discussions. Computers in Human Behavior, v. 55, p. 268-277, 2016.

ARISTEIDOU, M; SCANLON, E; SHARPLES, M. Profiles of engagement in online communities of citizen science participation. Computers in Human Behavior, v. 74, p. 246256, 2017.

BACQ, S.; GEOGHEGAN, W.; JOSEFY, M.; STEVENSON, R.; WILLIAMS, T. A. The COVID-19 Virtual Idea Blitz: Marshaling social entrepreneurship to rapidly respond to urgent grand challenges. Business Horizons, published ahead of print, 12 May, 2020. https://doi.org/10.1016/j.bushor.2020.05.002

BARDIN, L. Análise de Conteúdo. São Paulo, SP: Edições 70, 2011.

BODIN, Ö. Collaborative environmental governance: achieving collective action in socialecological systems. Science, v. 357, n. 6352, p. eaan1114, 2017.

BORTOLASO, I. V.; VERSCHOORE, J. R. Práticas de gestão de redes de cooperação horizontais: proposição de um modelo de análise. Contabilidade, Gestão e Governança, v. 16, n. 3, 2013.

BRABHAM, D. C. Crowdsourcing. Cambridge, MA: The MIT Press, 2013.

CANETTI, E. Crowds and Power. New York, NY: Farrar, Straus and Giroux, 1984.

COLLIS, J.; HUSSEY, R. Pesquisa em Administração. Porto Alegre, RS: Bookman, 2005.

CRESWELL, J. W. Qualitative Inquiry and Research Design: Choosing Among Five Traditions. Thousand Oaks, CA: Sage Publications, 1998.

CRISTOFOLI, D.; MACCIÒ, L.; PEDRAZZI, L. Structure, mechanisms, and managers in successful networks. Public Management Review, v. 17, n. 4, p. 489-516, 2015.

CROSS, R.; EHRLICH, K.; DAWSON, R.; HELFERICH, J. Managing collaboration: Improving team effectiveness through a network perspective. California Management Review, v. 50, n. 4, p. 74-98, 2008.

DARKE, P.; SHANKS, G.; BROADBENT, M. Successfully completing case study research: combining rigour, relevance and pragmatism. Information Systems Journal, v. 8, n. 4, p. 273-289, 1998.

(c) (1) 9 REAd | Porto Alegre - Vol. 26 - N. ${ }^{\circ} 2$ - Maio / Agosto 2020 - p. 238-264. 
EMERSON, K.; NABATCHI, T.; BALOGH, S. An integrative framework for collaborative governance. Journal of Public Administration Research and Theory, v. 22, n. 1, p. 1-29, 2012.

FERREIRA, G. D.; FARIAS, J. S. The motivation to participate in citizen-sourcing and hackathons in the public sector. BAR-Brazilian Administration Review, v. 15, n. 3, p. e180006, 2018.

FERREIRA, G. D.; FARIAS, J. S. Hackathons no setor público brasileiro: Objetivos e resultados sob a ótica de agentes públicos promotores das iniciativas. Contextus: Revista Contemporânea de Economia e Gestão, v. 17, n. 1, p. 195-216, 2019.

FOLHA DE SP. Médicos Relatam medo e angústia com avanço da pandemia de Covid19. 27/03/2020. https://www1.folha.uol.com.br/equilibrioesaude/2020/03/medicos-relatammedo-e-angustia-com-avanco-da-pandemia-de-covid-19.shtml. Acessado em 4 de agosto de 2020.

G1 PR. Hackaton que busca soluções para a vida pós-pandemia tem inscrições abertas. 17/04/2020. https://g1.globo.com/pr/parana/noticia/2020/04/17/hackaton-que-buscasolucoes-para-a-vida-pos-pandemia-tem-inscricoes-abertas.ghtml. Acessado em 4 de agosto de 2020.

GALTON, F. Vox Populi. Nature, v. 75, n. 1949, p. 450-451, 1907.

GARETTE, B.; DUSSAUGE, P. Alliances versus acquisitions: choosing the right option. European Management Journal, v. 18, n. 1, p. 63-69, 2000.

GLANCE, N.; HUBERMAN, B. A. The dynamics of social dilemmas. Scientific American, v. 270, n. 3, p. 76-81, 1994.

GRANDORI, A.; SODA, G. Inter-firm networks: antecedents, mechanisms and forms. Organization Studies, v. 16, n. 2, p. 183-214, 1995.

HARDT, M.; NEGRI, A. Multidão. Guerra e democracia na era do império. Rio de Janeiro, RJ: Record, 2005.

JOHNSON, P.; ROBINSON, P. J.; Civic hackathons: Innovation, procurement, or civic engagement?. Review of Policy Research, v. 31, n. 4, p. 349-357, 2014.

JORNAL DO COMÉRCIO. Rio Grande do Sul lidera maior hackathon on-line contra o $\begin{array}{llll}\text { coronavírus no 19/03/2020. } & \text { Brasil. }\end{array}$

https://www.jornaldocomercio.com/_conteudo/colunas/mercado_digital/2020/03/730380estado-lidera-maior-hackathon-online-do-combate-ao-coronavirus.html. Acessado em 4 de agosto de 2020.

KIENZLER, H.; FONTANESI, C. Learning through inquiry: A global health hackathon. Teaching in Higher Education, v. 22, n. 2, p. 129-142, 2017.

KIESLINGER, B.; PATA, K.; FABIAN, C. A participatory design approach for the support of collaborative learning and knowledge building in networked organizations. International Journal of Advanced Corporate Learning, v. 2, n. 3, p. 34-38, 2009.

KITTUR, A.; KRAUT, R. E. Harnessing the wisdom of crowds in wikipedia: quality through coordination. Proceedings of the 2008 ACM conference on Computer supported cooperative work, p. 37-46, 2008.

KRUEGER, R. A.; CASEY, M. A. Focus Groups: A practical guide for applied research. Thousand Oaks, CA: Sage Publications, 2008.

REAd | Porto Alegre - Vol. 26 - N. ${ }^{\text {o }} 2$ - Maio / Agosto 2020 - p. 238-264. 
LIFSHITZ-ASSAF, H.; LEBOVITZ, S.; ZALMANSON, L. Minimal and Adaptive Coordination: How Hackathons' Projects Accelerate Innovation without Killing it. Academy of Management Journal, published ahead of print, 5 May, 2020. https://doi.org/10.5465/amj.2017.0712

LIN, H. F. Effects of extrinsic and intrinsic motivation on employee knowledge sharing intentions, Journal of Information Science, v. 33 n. 2, p. 135-149, 2007.

LUZ, L. H. D. Estratégia emergente na lógica da multidão: uma abordagem a partir de iniciativas da multidão, do comum e da Teoria Ator-Rede. Tese de Doutorado. Universidade do Vale do Rio dos Sinos, 2016.

MALINEN, S. Understanding user participation in online communities: A systematic literature review of empirical studies. Computers in Human Behavior, v. 46, p. 228-238, 2015.

MARBACH, D.; COSTELLO, J. C.; KÜFFNER, R.; VEGA, N. V.; PRILL, R.J.; CAMACHO, D. M.; ALLISON, K. R.; KELLIS, M.; COLLINS, J. J.; STOLOVITZKY, G. Wisdom of crowds for robust gene network inference. Nature Methods, v. 9, n. 8, p. 796, 2012.

MARIANI, M. M. Coordination in inter-network co-opetitition: Evidence from the tourism sector. Industrial Marketing Management, v. 53, p. 103-123, 2016.

MICHELUCCI, P.; DICKINSON, J. L. The power of crowds. Science, v. 351 n. 6268, p. 3233, 2016.

MORETTI, A. The Network Organization: A Governance Perspective on Structure, Dynamics and Performance. London, UK: Palgrave Macmillan, 2017.

NOHRSTEDT, D.; BODIN, Ö. Collective action problem characteristics and partner uncertainty as drivers of social tie formation in collaborative networks. Policy Studies Journal, published ahead of print, 22 January, 2019. https://doi.org/10.1111/psj.12309

PADILHA, L. G. O.; VERSCHOORE, J. R. Green Governance: A proposal for collective governance constructs towards local sustainable development. Ambiente \& Sociedade, v. 16, n. 2, p. 153-174, 2013.

PAHOR, M.; ŠKERLAVAJ, M.; DIMOVSKI, V. Evidence for the network perspective on organizational learning. Journal of the American Society for Information Science and Technology, v. 59, n. 12, p. 1985-1994, 2008.

PEGN. Shawee fará competições de programação para achar soluções contra efeitos do coronavírus. 26/03/2020. https://revistapegn.globo.com/Startups/noticia/2020/03/shaweefara-competicoes-de-programacao-para-achar-solucoes-contra-efeitos-do-coronavirus.html.

Acessado em 4 de agosto de 2020.

PINTO, L. F. S.; SANTOS, C. D. Motivations of crowdsourcing contributors. Innovation \& Management Review, v. 15, n. 1, p. 58-72, 2018.

PROVAN, K. G.; MILWARD, H. B. A preliminary theory of interorganizational network effectiveness: A comparative study of four community mental health systems. Administrative Science Quarterly, v. 40, n. 1, p. 1-33, 1995.

PROVAN, K. G.; KENIS, P. Modes of network governance: Structure, management, and effectiveness. Journal of Public Administration Research and Theory, v. 18, n. 2, p. 229$252,2008$. 
RATTEN, V. Coronavirus (covid-19) and social value co-creation. International Journal of Sociology and Social Policy, published ahead of print, 14 July, 2020. https://doi.org/10.1108/IJSSP-06-2020-0237

ROBINSON, P. J.; JOHNSON, Peter A. Civic hackathons: New terrain for local governmentcitizen interaction?. Urban Planning, v. 1, n. 2, p. 65-74, 2016.

SAHIN, F.; ROBINSON, E. P. Flow coordination and information sharing in supply chains: review, implications, and directions for future research. Decision Sciences, v. 33, n. 4, p. 505536, 2002.

SCARSO, E.; BOLISANI, E. Communities of practice as structures for managing knowledge in networked corporations. Journal of Manufacturing Technology Management, v. 19, n. 3, p. 374-390, 2008.

SØRENSEN, E.; TORFING, J. The democratic anchorage of governance networks. Scandinavian Political Studies, v. 28, n. 3, p. 195-218, 2005.

TECHTUDO. Hack for Brazil: hackathon online busca diminuir impactos do coronavírus. 24/03/2020. https://www.techtudo.com.br/noticias/2020/03/hackathon-online-busca-diminuiros-impactos-do-coronavirus-no-brasil.ghtml. Acessado em 4 de agosto de 2020.

THERBORN, G. Back to Norms! On the Scope and Dynamics of Norms and Normative Action, Current Sociology, v. 50, n. 6, p. 863-880, 2002.

VENTURA, D. D. F. L.; RIBEIRO, H.; GIULIO, G. M. D.; JAIME, P. C.; NUNES, J.; BÓGUS, C. M.; ANTUNES, J. L. F.; WALDMAN, E. A. (2020). Desafios da pandemia de COVID-19: por uma agenda brasileira de pesquisa em saúde global e sustentabilidade. Cadernos de Saúde Pública, v. 36, n. 4, p. e00040620, 2020.

VERSCHOORE, J. R. El capital social y los nuevos instrumentos de políticas públicas para el desarrollo sostenido: la experiencia de Rio Grande do Sul, Brasil. Revista del CLAD Reforma y Democracia, n. 17, p. 127-160, 2000.

VIANNA, F. R. P. M.; MENEGHETTI, F. K. Is it crowdsourcing or crowdsensing? An analysis of human participation in digital platforms in the age of surveillance capitalism.

REAd - Revista Eletrônica de Administração, v. 26, n. 1, p. 210-243, 2020.

WILKINSON, D. M.; HUBERMAN, B. A. Cooperation and quality in wikipedia. Proceedings of the 2007 international symposium on Wikis, p. 157-164, 2007.

YASEEN, S. G.; AL OMOUSH, K. S. Mobile Crowdsourcing Technology Acceptance and Engagement in Crisis Management: The Case of Syrian Refugees. International Journal of Technology and Human Interaction, v. 16, n. 3, p. 1-23, 2020.

YIN, R. K. Estudo de Caso. Planejamento e Métodos. Porto Alegre, RS: Bookman, 2010.

YUAN, Q.; GASCO-HERNANDEZ, M. Open innovation in the public sector: creating public value through civic hackathons. Public Management Review, published ahead of print, 10 December, 2019. https://doi.org/10.1080/14719037.2019.1695884 